\title{
TWO NOTES ON LOCALLY MACAULAY RINGS
}

\author{
BY \\ LOUIS J. RATLIFF, JR.
}

1. Introduction. In this paper all rings are assumed to be commutative rings with a unit. The undefined terminology used in this paper (height, altitude, etc.) will be the same as that in [1]. Throughout this paper a number of known properties of locally Macaulay rings are stated, and then are used in the remainder of the paper without explicit mention.

In $\S 2$ it is proven that if $R$ is a locally Macaulay ring and if $\left(a_{1}, \cdots, a_{n}\right)$ is a prime sequence in $R$, the kernel of the natural homomorphism from $P=R\left[X_{1}, \cdots, X_{n-1}\right]$ onto $R^{\prime}=R\left[a_{2} / a_{1}, \cdots, a_{n} / a_{1}\right]$ is $\left(a_{1} X_{1}-a_{2}, a_{1} X_{2}-a_{3}, \cdots, a_{1} X_{n-1}-a_{n}\right) P$ (Lemma 2.3). As a consequence, $R^{\prime}$ is a locally Macaulay ring and $\left(a_{1}, a_{2} / a_{1}, \cdots, a_{n} / a_{1}\right)$ is a prime sequence in $R^{\prime}$ (Theorem 2.4). Further, if $R\left[X_{1}\right]$ is a Macaulay ring, then $R^{\prime}$ is a Macaulay ring (Theorem 2.8). An example is given to show that the converses are not in general true.

In $\$ 3$ it is proven that, with the same $R$ and $a_{i}$, the Rees ring $R^{*}$ $=R\left[t a_{1}, \cdots, t a_{n}, 1 / t\right](t$ an indeterminant $)$ of $R$ with respect to $A=\left(a_{1}, \cdots, a_{n}\right) R$ is a locally Macaulay ring (a Macaulay ring if $R\left[X_{1}\right]$ is) and $\left(1 / t, t a_{1}, \cdots, t a_{n}\right)$ is a prime sequence in $R^{*}$ (Theorems 3.1 and 3.3). A form of the converses of Theorems 3.1 and 3.3 is true (Theorem 3.8). Also, for every $e \geqq 1, k \geqq e$, and $i=1, \cdots, n$, $\left(a_{1}^{e}, a_{2}^{e}, \cdots, a_{i}^{e}\right) A^{k-e}=\left(a_{1}^{e}, a_{2}^{e}, \cdots, a_{i}^{e}\right) \cap A^{k}$ (Corollary 3.6). Further, for all $k \geqq 1$, every prime divisor of $A^{k}$ has height $n$, and $A^{k}: a_{1} R=A^{k-1}$ (Corollary 3.7). It is also proven that if the Rees ring $R^{*}$ of a Noetherian ring $R$ with respect to an ideal $A=\left(a_{1}, \cdots, a_{n}\right) R$ is a locally Macaulay ring (a Macaulay ring), then $R^{\prime}=R\left[a_{1} / a, \cdots, a_{n} / a\right]$ is a locally Macaulay ring (a Macaulay ring) for every nonzero-divisor $a \in A$ (Corollary 3.9).

\section{Transformations of locally Macaulay rings by a prime sequence.}

LEMMA 2.1. Let $R$ be a ring, let $a, b$ be elements in $R$ such that $a$ is not a zero divisor, and let $X$ be an indeterminant. If $a R: b R=a R$, then the kernel $K$ of the natural homomorphism from $R[X]$ onto $R[b / a]$ is generated by $a X-b$.

Proof. Clearly $a X-b \in K$. Let $f(X)=r_{n} X^{n}+\cdots+r_{0} \in K$. Then $r_{n} b^{n}+r_{n-1} a b^{n-1}+\cdots+r_{0} a^{n}=0$, so $r_{n} \in a R: b^{n} R=a R$, say $r_{n}=d a$. Since $a$ is not a zero divisor, $g(X)=\left(d b+r_{n-1}\right) X^{n-1}+r_{n-2} X^{n-2}+\cdots+r_{0} \in K$, and $f(X)=(a X-b) d X^{n-1}+g(X)$. Hence, by induction on $n, f(X) \in(a X-b) R[X]$, so $K$ is generated by $a X-b$, q.e.d.

Presented to the Society, November 14, 1964; received by the editors September 29, 1964. 
A local (Noetherian) ring $R$ is a Macaulay local ring in case there exists a system of parameters $\left(a_{1}, \cdots, a_{n}\right)$ in $R$ such that $a_{i}$ is not in any prime divisor of $\left(a_{1}, \cdots, a_{i-1}\right) R(i=1, \cdots, n)$. In particular $a_{1}$ is not a zero divisor. A Noetherian ring $R$ is a locally Macaulay ring in case $R_{M}$ is a Macaulay local ring for every maximal ideal $M$ in $R . R$ is a Macaulay ring in case $R$ is a locally Macaulay ring such that height $M=$ altitude $R$ for every maximal ideal $M$ in $R$. It is known that if $R$ is a Macaulay local ring of altitude $n$ and if $\left(a_{1}, \cdots, a_{k}\right)$ is a subset of a system of parameters in $R$, then $R /\left(a_{1}, \cdots, a_{k}\right) R$ is a Macaulay local ring of altitude $n-k$ [3, p. 397]. Also, $R$ is a locally Macaulay ring if and only if the following theorem (the unmixedness theorem) holds: If an ideal $A$ in $R$ is generated by $k$ elements and if height $A=k(k \geqq 0)$, then every prime divisor of $A$ has height $k$ $[1$, p. 85]. These two facts immediately imply that if $R$ is a locally Macaulay ring (a Macaulay ring) and if $A$ is an ideal in $R$ which is generated by $k$ elements and has height $k$, then $R / A$ is a locally Macualay ring (a Macaulay ring). Finally, it is known that if $X_{1}, \cdots, X_{n}$ are algebraically independent over a Noetherian ring $R$, then $R\left[X_{1}, \cdots, X_{n}\right]$ is a locally Macaulay ring if and only if $R$ is $[1, \mathrm{p} .86]$.

These facts are used in the proof of

COROLLARY 2.2. Let $R$ be a locally Macaulay ring, and let $a, b$ be elements in $R$ such that $a$ is not a zero divisor and $a R: b R=a R$. Then $R[b / a]$ is a locally Macaulay ring.

Proof. $R[X]$ is a locally Macaulay ring, and the kernel of the natural homomorphism from $R[X]$ onto $R[b / a]$ is generated by $a X-b$ (Lemma 2.1). Since $a X-b$ is not a zero divisor in $R[X], R[b / a]$ is a locally Macaulay ring, q.e.d.

Theorem 2.4 below generalizes the above corollary. To obtain the generalization the following definitions and lemma will be used.

An integral domain $R$ satisfies the altitude formula in case the following condition holds: If $R^{\prime}$ is an integral domain which is finitely generated over $R$, and if $p^{\prime}$ is a prime ideal in $R^{\prime}$, then height $p^{\prime}+\operatorname{trd}\left(R^{\prime} / p^{\prime}\right) /\left(R / p^{\prime} \cap R\right)=$ height $p^{\prime}$ $\cap R+\operatorname{trd} R^{\prime} \mid R$. It is known that if an integral domain $R$ is a homomorphic image of a locally Macaulay ring, then $R$ satisfies the altitude formula [1, p. 130].

If $R$ is a locally Macaulay ring, and if $p \subset q$ are prime ideals in $R$, then $R_{q}$ is a Macaulay local ring [1, p. 86], so height $p+$ height $q / p=$ height $q[3$, p. 399]. This fact will be used in the future without explicit mention.

A sequence $\left(a_{1}, \cdots, a_{n}\right)$ of nonunits in a Noetherian ring $R$ is a prime sequence in case $a_{1}$ is not a zero divisor, $\left(a_{1}, \cdots, a_{i}\right) R: a_{i+1} R=\left(a_{1}, \cdots, a_{i}\right) R(i=1, \cdots, n-1)$, and $\left(a_{1}, \cdots, a_{n}\right) R \neq R$. It is known that if $R$ is a semi-local ring, and if $\left(a_{1}, \cdots, a_{n}\right)$ is a prime sequence of elements in the Jacobson radical of $R$, then $\left(a_{\pi 1}, \cdots, a_{\pi n}\right)$ is a prime sequence for every permutation $\pi$ of $\{1, \cdots, n\}$ [3, pp. 394-395].

Lemma 2.3. Let $R$ be a locally Macaulay ring, let $\left(a_{1}, \cdots, a_{n}\right)$ be a prime sequence in $R$, and let $X_{1}, \cdots, X_{n-1}$ be algebraically independent over $R$. Then 
the kernel $K$ of the natural homomorphism $\phi$ from $P=R\left[X_{1}, \cdots, X_{n-1}^{-}\right]$onto $R^{\prime}=R\left[a_{2} / a_{1}, \cdots, a_{n} / a_{1}\right]$ is generated by $\left(a_{1} X_{1}-a_{2}, a_{1} X_{2}-a_{3}, \cdots, a_{1} X_{n-1}-a_{n}\right)$.

Proof( $\left.{ }^{1}\right)$. The proof is by induction on $n$. The case $n=1$ is trivial, and Lemma 2.1 proves the case $n=2$. Let $n>2$ and assume the conclusion holds for the case $n-1$. Now $\phi=f g$, where $f$ and $g$ are the natural homomorphisms from $S=R\left[a_{2} / a_{1}, X_{2}, \cdots, X_{n-1}\right]$ onto $R^{\prime}$ and from $P$ onto $S$ respectively. Since the kernel of $g$ is $\left(a_{1} X_{1}-a_{2}\right) P$ (Lemma 2.1), and since $R^{*}=R\left[a_{2} / a_{1}\right]$ is a locally Macaulay ring (Corollary 2.2), it is sufficient (by induction) to prove that $\left(a_{1}, a_{3}, a_{4}, \cdots, a_{n}\right)$ is a prime sequence in $R^{*}$. Since $R$ and $R^{*}$ have the same total quotient ring, $a_{1}$ is not a zero divisor in $R^{*}$, hence height $a_{1} R^{*}=1$. Let $A_{i}^{*}=\left(a_{1} X_{1}-a_{2}, a_{1}, a_{3}, \cdots, a_{i}\right) P \quad(i \geqq 3)$. Then $A_{i}^{*}=\left(a_{1}, a_{2}, a_{3}, \cdots, a_{i}\right) P$, hence height $A_{i}^{*}=i$. Consequently, by the unmixedness theorem $\left(a_{1} X_{1}-a_{2}, a_{1}, a_{3}, \cdots, a_{n}\right)$ is a prime sequence in $P$, hence $\left(a_{1}, a_{3}, \cdots, a_{n}\right)$ is a prime sequence in $R^{*}$, q.e.d.

THEOREM 2.4. With the same notation as Lemma 2.3, $R_{i}^{\prime}=R\left[a_{2} / a_{1}, \cdots, a_{i} / a_{1}\right]$ $(2 \leqq i \leqq n)$ is a locally Macaulay ring, and $\left(a_{1}, a_{i+1}, \cdots, a_{i+j}, b_{1}, \cdots, b_{k}\right)$ is $a$ prime sequence in $R_{i}^{\prime}$, where $\left\{b_{1}, \cdots, b_{k}\right\}$ is a subset of $\left\{a_{2} / a_{1}, \cdots, a_{i} / a_{1}\right\}$, and $0 \leqq j \leqq n-i$. (For $j=0$ the sequence is $\left(a_{1}, b_{1}, \cdots, b_{k}\right)$.)

Proof. That $R_{i}^{\prime}$ is a locally Macaulay ring follows immediately from Lemma 2.3 and the remarks preceding the proof of Corollary 2.2. Let $A^{*}=\left(a_{1}, a_{i+1}, \cdots, a_{i+j}\right.$, $\left.b_{1}, \cdots, b_{k}\right) R_{i}^{\prime}$. Since $\left(a_{1} X_{1}-a_{2}, \cdots, a_{1} X_{i-1}-a_{i}, a_{1}\right) R\left[X_{1}, \cdots, X_{i-1}\right]$ is generated by $\left(a_{1}, \cdots, a_{i}\right), A^{*}$ is a proper ideal. Hence by the unmixedness theorem, since $j$ and $k$ are arbitrary, it is sufficient to prove height $A^{*}=j+k+1$. Let $p^{\prime}$ be a minimal prime divisor of $A^{*}$, let $q^{\prime}$ be a (minimal) prime divisor of zero in $R_{i}^{\prime}$ such that $q^{\prime} \subset p^{\prime}$ and let $p=p^{\prime} \cap R, q=q^{\prime} \cap R$. By the altitude formula (for $R^{\prime} / q^{\prime}$ over $R / q$ ), height $p^{\prime} / q^{\prime}+\operatorname{trd} R^{\prime} / p^{\prime} / R / p=$ height $p / q$ (since $a_{1} \notin q$ ). Also, height $p^{\prime} / q^{\prime} \leqq j+k+1, \operatorname{trd} R^{\prime} / p^{\prime} \mid R / p \leqq i-1-k$, and height $p / q=$ height $p \geqq i+j$. Hence, height $p^{\prime}=$ height $p^{\prime} / q^{\prime}=j+k+1$. Therefore height $A^{*}=j+k+1$, q.e.d.

REMARK 2.5. The last step in the proof of Theorem 2.4 shows the following results. For every (minimal) prime divisor $p^{\prime}$ of $A^{*}$ and for every prime divisor $q^{\prime}$ of zero contained in $p^{\prime}, p^{\prime} / q^{\prime}$ is a minimal prime divisor of $\left(A^{*}+q^{\prime}\right) / q^{\prime}$. Since height $p^{\prime} / q^{\prime}=j+k+1$, none of the elements $a_{1}, \cdots, a_{i+j}, b_{1}, \cdots, b_{k}$ are in $q^{\prime}$. Also the elements $a_{2} / a_{1}, \cdots, a_{i} / a_{1}$ which are not in $p^{\prime}$ are such that their $p^{\prime}$ residues are algebraically independent over $R /\left(p^{\prime} \cap R\right)$.

REMARK 2.6. In Theorem 2.4, if every permutation of $\left(a_{1}, \cdots, a_{n}\right)$ is a prime sequence in $R$ (for example, if $R$ is a semi-local locally Macaulay ring and $a_{1}, \cdots, a_{n}$ are in the Jacobson radical of $R)$, then every permutation of $\left(a_{1}, a_{i+1}, \cdots, a_{n}\right.$, $\left.a_{2} / a_{1}, \cdots, a_{i} / a_{1}\right)$ is a prime sequence in $R_{i}^{\prime}$.

(1) The author is indebted to the referee for the following proof which is considerably simpler than the author's original proof, and which leads to a more direct proof of Theorem 2.4 . 
Proof. Let $\left(c_{1}, \cdots, c_{n}\right)$ be a permutation of $\left(a_{1}, a_{i+1}, \cdots, a_{n}, a_{2} / a_{1}, \cdots, a_{i} / a_{1}\right)$. Since no $a_{i}$ is a zero divisor in $R, c_{1}$ is not a zero divisor in $R_{i}^{\prime}$. Also $\left(c_{1}, \cdots, c_{n}\right) R_{i}^{\prime} \neq R_{i}^{\prime}$. Therefore, by the unmixedness theorem, it remains to prove height $\left(c_{1}, \cdots, c_{h}\right) R_{i}^{\prime}=h$ $(h=2, \cdots, n-1)$. Let $p^{\prime}$ be a minimal prime divisor of $\left(c_{1}, \cdots, c_{h}\right) R_{i}^{\prime}$, let $q^{\prime}$ be a prime divisor of zero in $R_{i}^{\prime}$ which is contained in $p^{\prime}$, and let $p=p^{\prime} \cap R, q=q^{\prime} \cap R$. If $a_{1} \notin p^{\prime}$, then $\operatorname{trd} R^{\prime} / p^{\prime} \mid R / p=0$. Hence by the altitude formula (for $R^{\prime} / q^{\prime}$ over $R / q$ ), height $p^{\prime} / q^{\prime}=$ height $p / q$. Now height $p^{\prime} \leqq h$ and height $p \geqq h$ (by the assumption on $\left(a_{1}, \cdots, a_{n}\right)$ ), so height $p^{\prime}=$ height $p=h$. If $a_{1} \in p^{\prime}$, let $k$ of the elements $c_{1}, \cdots, c_{h}$ be in $\left\{a_{2} / a_{1}, \cdots, a_{i} / a_{1}\right\}$. Then height $p \geqq i+(h-1-k)$ (by the assumption on $\left.\left(a_{1}, \cdots, a_{n}\right)\right)$, and $\operatorname{trd} R^{\prime} / p^{\prime} \mid R / p \leqq i-1-k$. By the altitude formula for $R^{\prime} / q^{\prime}$ over $R / q$, height $p^{\prime}=$ height $p^{\prime} / q^{\prime}=h$, q.e.d.

Remark 2.6 is of some interest because of the following

Lemma 2.7. Let $R$ be a locally Macaulay ring, and let $\left(a_{1}, \cdots, a_{n}\right)$ be a prime sequence in $R$ such that every permutation of $\left(a_{1}, \cdots, a_{n}\right)$ is a prime sequence in $R$. Let $A=\left(a_{1}, \cdots, a_{n}\right) R$. Then, for all $k \geqq 1$, (1) every prime divisor of $A^{k}$ has height $n$, and (2) $A^{k}: a_{i} R=A^{k-1}(i=1, \cdots, n)$.

Proof. This can be proved in the same way as Lemmas 5 and 6 in [3, pp. 401-402]. Without assuming that every permutation of $\left(a_{1}, \cdots, a_{n}\right)$ is a prime sequence in $R$, Corollary 3.7 below proves (1) is still true and (2) $A^{k}: a_{1} R=A^{k-1}$ (for all $k \geqq 1$ ), q.e.d.

It is known that if $R$ is a Macaulay ring and if $X_{1}, \cdots, X_{n}$ are algebraically independent over $R$, then $R\left[X_{1}, \cdots, X_{n}\right]$ is a Macaulay ring if and only if there does not exist an ideal $p$ in $R$ such that $R / p$ is a semi-local integral domain of altitude one [1, p. 87]. Hence if $R\left[X_{1}\right]$ is a Macaulay ring, then $R\left[X_{1}, \cdots, X_{n}\right]$ is a Macaulay ring. This fact is used in the proof of the next theorem.

THEOREM 2.8. If $R$ and $R[X]$ are Macaulay rings ( $X$ transcendental over $R$ ), and if $\left(a_{1}, \cdots, a_{n}\right)$ is a prime sequence in $R$, then $R^{\prime}=R\left[a_{2} / a_{1}, \cdots, a_{n} / a_{1}\right]$ is $a$ Macaulay ring.

Proof. The kernel $K$ of the natural homomorphisms from $P=R\left[X_{1}, \cdots, X_{n-1}\right]$ onto $R^{\prime}$ has height $n-1$. Since $P$ is a Macaulay ring, if $M$ is a maximal ideal in $P$ which contains $K$, then altitude $R+n-1=$ altitude $P=$ height $M=$ height $M / K+$ height $K$. Hence, if $M^{\prime}$ is a maximal ideal in $R^{\prime}$, then height $M^{\prime}=$ altitude $P$ - height $K=$ altitude $R$. Since $R^{\prime}$ is a locally Macaulay ring by Theorem $2.4, R^{\prime}$ is a Macaulay ring, q.e.d.

REMARK 2.9. If $R$ is a locally Macaulay ring (a Macaulay ring such that $R[X]$ is a Macaulay ring), and if $\left(a_{1}, \cdots, a_{n}\right)$ is a prime sequence in $R$, then $R\left[a_{1} / a, \cdots, a_{n} / a\right]$ is a locally Macaulay ring (a Macaulay ring) for every non-zero-divisor $a \in\left(a_{1}, \cdots, a_{n}\right) R$. This follows from Theorems 3.1 and 3.3 and Corollary 3.9 below. It will now be shown that the converses of Theorems 2.4 and 2.8 are not in 
general true. Let $S=k[X, Y]$, where $k$ is a field and $X$ and $Y$ are algebraically independent over $k$. Let $P=(X-1, Y) S, R_{1}=S_{P}$, and $N_{1}=P R_{1}$. Let $Q=(X, Y) S, R_{2}=S_{Q}$, and $N_{2}=Q R_{2}$. Let $R^{\prime}=R_{1} \cap R_{2}, M_{1}=N_{1} \cap R^{\prime}$, and $M_{2}=N_{2} \cap R^{\prime}$. Further let $R=k+\left(M_{1} \cap M_{2}\right)$, and let $M=\left(M_{1} \cap M_{2}\right) R$. Then $R^{\prime}$ is the intersection of two regular local rings, hence $R^{\prime}$ is normal. The following statements are easily verified: (1) $M_{1}$ and $M_{2}$ are the maximal ideals in $R^{\prime}$, and $R_{M_{i}}^{\prime}=R_{i}$ is Noetherian $(i=1,2)$. Therefore $R^{\prime}$ is Noetherian [1, p. 203], so $R^{\prime}$ is a normal semi-local Macaulay domain. (2) Since $R^{\prime} / M_{i}=k(i=1,2)$, $R$ is a local domain and $R^{\prime}$ is its derived normal ring [1, p. 204]. (3) $X Y, Y \in R$, $X \notin R, R^{\prime}=R[X Y / Y]$, and $(Y, X=X Y / Y)$ is a prime sequence in $R^{\prime}$. (4) If $p$ is a height one prime ideal in $R$, then $R_{p}$ is a regular local ring. Since $R \neq R^{\prime}, M$ is an imbedded prime divisor of every nonzero element in $M[1, \mathrm{p} .41]$, hence $R$ is not a Macaulay domain.

3. The Rees ring of a locally Macaulay ring. Let $R$ be a Noetherian ring, let $A=\left(a_{1}, \cdots, a_{n}\right) R$ be an ideal in $R$, let $t$ be an indeterminant, and set $u=t^{-1}$. The graded Noetherian ring $R^{*}=R\left[t a_{1}, \cdots, t a_{n}, u\right]$ is called the Rees ring of $R$ with respect to $A$.

THEOREM 3.1. Let $R$ be a locally Macaulay ring, and let $a_{1}, \cdots, a_{n}$ be a prime sequence in $R$. Then the Rees ring $R_{i}^{*}$ of $R$ with respect to $\left(a_{1}, \cdots, a_{i}\right) R(1 \leqq i \leqq n)$ is a locally Macaulay ring, and $\left(u, a_{i+1}, \cdots, a_{i+j}, b_{1}, \cdots, b_{k}\right)$ is a prime sequence in $R_{i}^{*}$, where $\left\{b_{1}, \cdots, b_{k}\right\}$ is a subset of $\left\{t a_{1}, \cdots, t a_{i}\right\}$ and $0 \leqq j \leqq n-i$. (For $j=0$ the sequence is $\left(u, b_{1}, \cdots, b_{k}\right)$.)

Proof. Since $u$ is transcendental over $R, R[u]$ is a locally Macaulay ring, hence $\left(u, a_{1}, \cdots, a_{n}\right)$ is a prime sequence in $R[u]$. Since $t a_{j}=a_{j} / u, R_{i}^{*}$ is a locally Macaulay ring and $\left(u, a_{i+1}, \cdots, a_{i+j}, b_{1}, \cdots, b_{k}\right)$ is a prime sequence in $R_{i}^{*}$ by Theorem 2.4, q.e.d.

REMARK 3.2. In Theorem 3.1 , if every permutation of $\left(a_{1}, \cdots, a_{n}\right)$ is a prime sequence in $R$, then every permutation of $\left(u, a_{1}, \cdots, a_{n}\right)$ is a prime sequence in $R[u]$ (since $R[u]$ is a locally Macaulay ring and $u$ is transcendental over $R$ ), hence by Remark 2.6 every permutation of $\left(u, a_{i+1}, \cdots, a_{n}, t a_{1}, \cdots, t a_{i}\right)$ is a prime sequence in $R_{i}^{*}$.

THEOREM 3.3. If $R$ and $R[X]$ are Macaulay rings ( $X$ transcendental over $R$ ), and if $a_{1}, \cdots, a_{n}$ is a prime sequence in $R$, then the Rees ring $R^{*}$ of $R$ with respect to $\left(a_{1}, \cdots, a_{n}\right) R$ is a Macaulay ring.

Proof. Considering the natural homomorphism from $R\left[u, X_{1}, \cdots, X_{n}\right]$ onto $R^{*}$ and the ideal $\left(u, a_{1}, \cdots, a_{n}\right)$ of $R^{*}$, the proof is the same as the proof of Theorem 2.8 , q.e.d.

LEMMA 3.4. Let $R^{*}$ be the Rees ring of a locally Macaulay ring $R$ with respect to a prime sequence $\left(a_{1}, \cdots, a_{n}\right)$ in $R$. Then $\left(t a_{1}, \cdots, t a_{i}, u\right)$ is a prime sequence in $R^{*}(i=1, \cdots, n)$. 
Proof. Since $R^{*}$ is a locally Macaulay ring and height $\left(u, t a_{1}, \cdots, t a_{i}\right) R^{*}=i+1$ (Theorem 3.1), it is sufficient to prove height $\left(t a_{1}, \cdots, t a_{i}\right) R^{*}=i$. Let $p$ be a minimal prime divisor of $A_{i}^{*}=\left(t a_{1}, \cdots, t a_{i}\right) R^{*}$. Then height $p \leqq i$, hence $u \notin p$. Let $T=R[u, t]$, so $T$ is a quotient ring of $R^{*}$. Since $p T$ is a minimal prime divisor of $A_{i}^{*} T=\left(a_{1}, \cdots, a_{i}\right) T$, and since height $\left(a_{1}, \cdots, a_{i}\right) R[u]=i$, height $A_{i}^{*} T=i$. Therefore height $p=i$, so height $A_{i}^{*}=i$, q.e.d.

RemarK 3.5. Let $\left(a_{1}, \cdots, a_{n}\right)$ be a prime sequence in a locally Macaulay ring $R$. Then the radical of $\left(a_{1}, \cdots, a_{n}\right) R$ is the radical of $\left(a_{1}^{e_{1}}, a_{2}^{e_{2}}, \cdots, a_{n}^{e_{n}}\right) R\left(e_{i} \geqq 1, i=1, \cdots, n\right)$. Hence, by the unmixedness theorem, $\left(a_{1}^{e_{1}}, a_{2}^{e_{2}}, \cdots, a_{n}^{e_{n}}\right)$ is a prime sequence in $R$. Therefore $R\left[a_{2}^{e_{2}} / a_{1}^{e_{1}}, \cdots, a_{n}^{e_{n}} / a_{1}^{e_{1}}\right]$ and $R\left[t a_{1}^{e_{1}}, \cdots, t a_{n}^{e_{n}}, u\right]$ are locally Macaulay rings.

Let $R$ be a Noetherian ring and let $R^{*}$ be the Rees ring of $R$ with respect to an ideal $A$ in $R$. Let $T=R[t, u]$, so $T$ is a quotient ring of $R^{*}$. For any ideal $B$ in $R$ let $B^{\prime}=B T \cap R^{*}$. For any homogeneous ideal $B^{*}$ in $R^{*}$ let $\left[B^{*}\right]_{k}$ be the set of elements $r \in R$ such that $r t^{k} \in B^{*}$. It is immediately seen that $\left[B^{*}\right]_{k}$ is an ideal in $R$ and $A^{k} \supseteq\left[B^{*}\right]_{k} \supseteq\left[B^{*}\right]_{k+1} \supseteq A\left[B^{*}\right]_{k}$ for all integers $k$ (with the convention that $A^{k}=R$ if $k \leqq 0$ ). Also, since $R^{*}$ is Noetherian, if $k$ is greater than or equal to the maximum degree of the generators of $B^{*}$, then $\left[B^{*}\right]_{k+1}=A\left[B^{*}\right]_{k}$, and if $k$ is less than or equal to the degree of the generators of $B^{*}$, then $\left[B^{*}\right]_{k-1}=\left[B^{*}\right]_{k}[2]$. Let $B=\left(b_{1}, \cdots, b_{i}\right) R \subseteq A^{e}$. Clearly $B^{\prime}=B T \cap R^{*} \supseteq\left(b_{1} t^{e}, b_{2} t^{e}, \cdots, b_{i} t^{e}\right) R^{*}=B^{*}$, and for $k \leqq e,\left[B^{\prime}\right]_{k}=B \cap A^{k}=B \supseteq\left[B^{*}\right]_{k}=[B]_{e} \supseteq B$. Hence for $k>e$, $\left[B^{\prime}\right]_{k}=B \cap A^{k} \supseteq\left[B^{*}\right]_{k}=B A^{k-e}$. Since $B^{\prime} T=B^{*} T=B T, B^{*}=B^{\prime}$ if and only if $u$ is not in any prime divisor of $B^{*}$. Hence if $\left(b_{1} t^{e}, b_{2} t^{e}, \cdots, b_{i} t^{e}, u\right)$ is a prime sequence in $R^{*}$, then $B^{\prime}=B^{*}$. In particular, by Lemma 3.4 and Remark 3.5 we have proved the following

COROllaRY 3.6. Let $R$ be a locally Macaulay ring, let $\left(a_{1}, \cdots, a_{n}\right)$ be a prime sequence, and let $A=\left(a_{1}, \cdots, a_{n}\right) R$. Then, for every $e \geqq 1, k \geqq e$, and $i=1, \cdots, n$, $\left(a_{1}^{e}, a_{2}^{e}, \cdots, a_{i}^{e}\right) A^{k-e}=\left(a_{1}^{e}, a_{2}^{e}, \cdots, a_{i}^{e}\right) R \cap A^{k}$.

COROLLARY 3.7. Let $\left(a_{1}, \cdots, a_{n}\right)$ be a prime sequence in a locally Macaulay ring R. Set $A=\left(a_{1}, \cdots, a_{n}\right) R$. Then, for all $k \geqq 1$, (1) every prime divisor of $A^{k}$ has height $n$, and (2) $A^{k}: a_{1} R=A^{k-1}$.

Proof. By Corollary 3.6, $a_{1} A^{k-1}=a_{1} R \cap A^{k}$. Since $a_{1}$ is not a zero divisor in $R, A^{k-1}=a_{1} A^{k-1}: a_{1} R=\left(a_{1} R \cap A^{k}\right): a_{1} R=A^{k}: a_{1} R$, hence (2) holds. For (1), $u^{k} R^{*} \cap R=A^{k}$, where $R^{*}=R\left[t a_{1}, \cdots, t a_{n}, u\right]$, and $k \geqq 1$. Since $R^{*}$ is a locally Macaulay ring, every prime divisor of $u R^{*}$ has height one, and the prime divisors of $u^{k} R^{*}$ are the prime divisors of $u R^{*}$ (Remark 3.5). Let $p^{\prime}$ be a prime divisor of $u R^{*}$, let $q^{\prime}$ be a minimal prime divisor of zero in $R^{*}$ which is contained in $p^{\prime}$, and let $p=p^{\prime} \cap R, q=q^{\prime} \cap R$. Applying Remark 2.5 (with $A^{*}=u R^{*}$ ) and the altitude formula for $R^{*} / q^{\prime}$ over $R / q$, height $p=n$ (since $\left.\operatorname{trd} R^{*} / q^{\prime} / R / q=1\right)$, so $p$ is a prime divisor of $A^{k}$. Since $u^{k} R^{*} \cap R=A^{k}$, (1) holds, q.e.d. 
If $\left(a_{1}, \cdots, a_{n}\right)$ is a prime sequence in a locally Macaulay ring $R$, then $\left(t a_{1}, \cdots, t a_{n}, u\right)$ is a prime sequence in the locally Macaulay ring $R\left[t a_{1}, \cdots, t a_{n}, u\right]$ (Theorem 3.1 and Lemma 3.4). Theorem 3.8 contains the converse of this.

THEOREM 3.8. Let $R$ be a Noetherian ring and let $A$ be an ideal in $R$. If the Rees ring $R^{*}$ of $R$ with respect to $A$ is a locally Macaulay ring (a Macaulay ring), then $R$ is a locally Macaulay ring ( $R$ and $R[X]$ are Macaulay rings). If also there are elements $b_{1}, \cdots, b_{n}$ in $A$ such that $\left(b_{1} t^{e_{1}}, \cdots, b_{n} t^{e_{n}}, u\right)$ is a prime sequence in $R^{*}$, then $\left(b_{1}, \cdots, b_{n}\right)$ is a prime sequence in $R$.

Proof. Let $R^{*}$ be a locally Macaulay ring. Then, since $T=R^{*}[t]$ is a quotient ring of $R^{*}, T$ is a locally Macaulay ring. Let $M$ be a maximal ideal in $R$. Since $T$ is a quotient ring of $R^{*}$ and of $R[u], T_{M T}=R[u]_{M R[u]}$ is a Macaulay local ring. Since $u$ is transcendental over $R$, a system of parameters in $R_{M}$ is a system of parameters in $R[u]_{M R[u]}$. It is known that if a local ring has one system of parameters which form a prime sequence, then each system of parameters forms a prime sequence [3, p. 399]. Hence $R$ is a locally Macaulay ring. Therefore, if $\left(b_{1} t^{e_{1}}, \cdots, b_{n} t^{e_{n}}, u\right)$ is a prime sequence in $R^{*}$, then, for $i=1, \cdots, n$, every prime divisor of $\left(b_{1} t^{e_{1}}, \cdots, b_{i} t^{e_{i}}\right) T=\left(b_{1}, \cdots, b_{i}\right) T$ has height $i$. Hence height $\left(b_{1}, \cdots, b_{i}\right) R=i$, and so $\left(b_{1}, \cdots, b_{n}\right)$ is a prime sequence in $R$. Let $R^{*}$ be a Macaulay ring. By what has already been proved, $R$ and $R[X]$ are locally Macaulay rings. To prove that $R$ is a Macaulay ring, let $M$ be a maximal ideal in $R$. Then $N^{*}=(M, u-1) T \cap R^{*}$ is a maximal ideal in $R^{*}$. Therefore, altitude $R+1=$ altitude $R^{*}=$ height $N^{*}=$ height $N^{*} T=$ height $M+1$, hence $R$ is a Macaulay ring. Finally, let $N$ be a maximal ideal in $R[u]$. If there is a maximal ideal $N^{*}$ in $R^{*}$ such that $N^{*} \cap R[u]=N$, then altitude $R[u]=$ altitude $R^{*}=$ height $N^{*}=$ (since $R^{*} / N^{*}$ is a field) height $N^{*}+$ $\operatorname{trd} R^{*} / N^{*} / R[u] / N=$ (altitude formula) height $N \leqq$ altitude $R[u]$. If there does not exist such $N^{*}$, then $N T=T$, hence $u \in N$. Therefore $R / N \cap R=R[u] / N$ is a field, so altitude $R[u]=$ altitude $R+1=$ height $N \cap R+1=$ height $N^{*}$. Hence $R[X] \cong R[u]$ is a Macaulay ring, q.e.d.

COROllary 3.9. Let $R$ be a Noetherian ring. If there exists an ideal $A=\left(a_{1}, \cdots, a_{n}\right) R$ in $R$ such that the Rees ring $R^{*}$ of $R$ with respect to $A$ is $a$ locally Macaulay ring (a Macaulay ring), then for every non-zero-divisor $a \in A, R^{\prime}=R\left[a_{1} / a, \cdots, a_{n} / a\right]$ is a locally Macaulay ring (a Macaulay ring).

Proof. Since $(a-u) R[t, u]=(a t-1) R[t, u]$ is the kernel of the mapping from $R[t, u]$ onto $R[1 / a, a]$ (Lemma 2.1), and since $R[t, u]$ is a quotient ring of $R^{*}$, to prove the two statements about $R^{\prime}$ it is sufficient to prove that $u$ is not in any prime divisor of $(t a-1) R^{*}$. If $u$ is in some (minimal) prime divisor $p$ of $(t a-1) R^{*}$, then $p$ is a prime divisor of $u R^{*}$. But $u R^{*}$ is a graded ideal, hence $p$ is a graded deal. This implies the contradiction $1 \in p$. Therefore $u$ is not in any prime divisor of $(t a-1) R^{*}$, q.e.d. 
Theorem 3.8 is of some interest, since the Rees ring $R^{*}$ of a locally Macaulay ring $R$ with respect to an ideal $A$ which cannot be generated by a prime sequence may be a locally Macaulay ring. For example, let $R$ be a semi-local Macaulay ring of altitude $n \geqq 2$, and let $\left(a_{1}, \cdots, a_{n}\right)$ be a prime sequence in the Jacobson radical of $R$. Let $A=\left(a_{1}, \cdots, a_{n}\right) R$ and fix an integer $e \geqq 2$. Then $A^{e}$ cannot be generated by $n$ elements, but the Rees ring of $R$ with respect to $A^{e}$ is a locally Macaulay ring. For convenience of notation this will be proved for the case $n=2$ (the general case being exactly the same). Let $a=a_{1}$ and $b=a_{2}$, and let $N$ be a maximal ideal in $R^{*}=R\left[t a^{e}, \cdots, t a^{f} b^{e-f}, \cdots, t b^{e}, u\right]$. If $u \notin N$, then $R_{N}^{*}$ contains $T=R[t, u]$. Since $T$ is a locally Macaulay ring, $R_{N}^{*}$ is a Macaulay local ring. If $\left(t a^{e}, \cdots, t a^{f} b^{e-f}, \cdots, t b^{e}\right) R^{*}$ is not contained in $N$, say $t a^{f} b^{e-f} \notin N$. Then $t a^{f+1} b^{e-f-1} / t a^{f} b^{e-f}=a / b \in R_{N}^{*}$ (if $f<e$ ), and/or $b / a \in R_{N}^{*}$ (if $f>0$ ). Since $(a, b)$ and $(b, a)$ are prime sequences in $R, R_{e}=R[a / b], R_{0}=R[b / a]$, and $R_{f}=R[a / b, b / a]$ are locally Macaulay rings, and at least one of these rings (call it $R^{\prime}$ ) is contained in $R_{N}^{*}$. Hence $S=R^{\prime}\left[t a^{f} b^{e-f}\right]$ is a locally Macaulay ring contained in $R_{N}^{*}$, and $S$ contains $R\left[t a^{e}, \cdots, t a^{g} b^{e-g}, \cdots, t b^{e}\right]$. Since $t a^{f} b^{e-f} \notin N^{\prime}$ $=N R_{N}^{*} \cap S, u=a^{f} b^{e-f} / t a^{f} b^{e-f} \in S_{N^{\prime}}$. Hence $R_{N}^{*}=S_{N^{\prime}}$ is a locally Macaulay ring. Clearly the only maximal ideals in $R^{*}$ which contain $\left(t a^{e}, \cdots, t a^{f} b^{e-f}, \cdots, t b^{e}, u\right) R^{*}$ are the ideals $N_{i}=\left(M_{i}, t a, \cdots, t a^{f} b^{e-f}, \cdots, t b^{e}, u\right) R^{*}$, where $M_{i}$ is a maximal ideal in $R$. Therefore it remains to prove that the semi-local ring $R_{R^{*}-\cup N_{i}}$ is a Macaulay ring. For this, it will be shown that $\left(t a^{e}, t b^{e}, u\right)$ is a prime sequence in $R^{*}$ (since the $N_{i}$ contain this sequence). Since $\left(a^{e}, b^{e}\right)$ is a prime sequence in the locally Macaulay ring $R^{*}[t]$, to prove $\left(t a^{e}, t b^{e}, u\right)$ is a prime sequence, it is sufficient to prove that $u$ is not in any prime divisor of either of the ideals $t a^{e} R^{*}$ or $\left(t a^{e}, t b^{e}\right) R^{*}$. This is equivalent to proving $t a^{e} R^{*}=a^{e} T \cap R^{*}$ and $\left(t a^{e}, t b^{e}\right) R^{*}=\left(a^{e}, b^{e}\right) T \cap R^{*}$, where $T=R[t, u]$. With the notation used in the proof of Corollary 3.6, these latter equalities are equivalent to $\left[t a^{e} R^{*}\right]_{k}=\left[a^{e} T \cap R^{*}\right]_{k}$ and $\left[\left(t a^{e}, t b^{e}\right) R^{*}\right]_{k}$ $=\left[\left(a^{e}, b^{e}\right) T \cap R^{*}\right]_{k}$ for all $k$. Since the degrees of the generators of the four ideals are all non-negative, and since $\left[t a^{e} R^{*}\right]_{0}=\left[a^{e} T \cap R^{*}\right]_{0}=a^{e} R$ and $\left[\left(t a^{e}, t b^{e}\right) R^{*}\right]_{0}=\left[\left(a^{e}, b^{e}\right) T \cap R^{*}\right]_{0}=\left(a^{e}, b^{e}\right) R$, it must be shown that $a^{e}\left(A^{e}\right)^{k-1}$ $=a^{e} R \cap\left(A^{e}\right)^{k}$ and $\left(a^{e}, b^{e}\right)\left(A^{e}\right)^{k-1}=\left(a^{e}, b^{e}\right) R \cap\left(A^{e}\right)^{k}$ for all $k \geqq 1$. These equalities hold by Corollary 3.6.

\section{REFERENCES}

1. M. Nagata, Local rings, Interscience, New York, 1962.

2. D. Rees, A-transforms of local rings and a theorem on multiplicities of ideals, Proc. Cambridge Philos. Soc. 57 (1961), 8-17.

3. O. Zariski and P. Samuel, Commutative algebra, Vol. II, Van Nostrand, Princeton, N. J., 1960.

UNIVERSITY OF CALIFORNIA, Riverside, CALIfornia 